\title{
Study of the Protective Effects of Quince (Cydonia Oblonga) Leaf Extract on the Histologic Structure and Microscopic Indices of Spermatogenesis Following Induction of Diabetes in Adult Rats
}

\author{
Davoud KIANIFARD ${ }^{1 *}$, Gholamreza VAFAEI SAIAH ${ }^{2}$, Emad KHALILZADEH ${ }^{2}$ \\ ${ }^{1}$ Division of Histology, Department of Basic Sciences, Faculty of Veterinary Medicine, University of \\ Tabriz, Tabriz, IRAN \\ ${ }^{2}$ Division of Physiology \& Laboratory Animals Facility, Department of Basic Sciences, Faculty of \\ Veterinary Medicine, University of Tabriz, Tabriz, IRAN \\ * corresponding author: davoudkianifard@gmail.com
}

Bulletin UASVM Veterinary Medicine 73(2)/2016

Print ISSN 1843-5270; Electronic ISSN 1843-5378

doi:10.15835/buasvmcn-vm:12301

\begin{abstract}
Various types of infertility are associated with uncontrolled hyperglycemia. Development of oxidative stress is one of the most important factors in the alteration of spermatogenesis in diabetic conditions. Testicular tissue is one of the sensitive organs to environmental damages. Natural antioxidants are considered as preventive and therapeutic strategies in cases of diabetic side effects. Cydonia oblonga leaf extract contains natural antioxidant compounds. The flavonoid compounds of the quince have strong antioxidant and immune-regulatory effects. According to little data about the protective effects of cydonia oblonga leaf extract on the structural alterations of testicular tissue following induction of diabetes, in this study protective aspects of this extract on the diabetic reproductive alteration were evaluated.

Cydonia oblonga leaf extract was gavaged in two doses of 250 and $500 \mathrm{mg} / \mathrm{kg}$. The number of 56 adult rats were divided into seven groups consisted of control, extract treated control groups, diabetic, extract treated diabetic groups and metformin treated diabetic group. Eight weeks after induction of diabetes, the body and testicular weight were measured and microscopic and histomorphometric studies were done on tissue samples.

In control groups, the administration of extract had no effect on histomorphometric parameters. In extract treated diabetic groups, the mean of body weight, histologic parameters and spermatogenesis indices were improved in comparison to non-treated diabetic group.

The results of this study showed that, natural antioxidant such as cydonia oblonga leaf extract in certain doses could be effective in reduction of hyperglycemic side effects on target organs.
\end{abstract}

Keywords: Cydonia Oblonga Extract, Diabetes, Rat, Spermatogenesis, Testicular tissue

\section{INTRODUCTION}

The acute and chronic complications of diabetes are the major problems that occur in diabetic patients (Yanardag et al., 2005). Increase of blood glucose levels leads to structural and functional changes in various target tissues and organs (Cai et al., 2000). Induction of diabetes in male rats is associated with alteration in functions of reproductive system (Orth et al., 1979). Diabetes is one of the most important risk factors for male infertility. In this regard, in vivo experiments on adult rats indicated that, induction of diabetes is associated with cellular alterations in testicular microenvironment (Kianifard et al., 2011).

Diabetes is a condition of increased oxidative stress and impaired energy metabolism. Recent experimental and clinical studies suggest that oxidative stress and production of reactive oxygen species (ROS) play a key role in the pathogenesis of both types of diabetes mellitus and subsequently 
development of diabetes complications (Maritim et al., 2003; Giacco and Brownlee, 2010). Herbal drugs have gained importance because of their efficacy and cost effectiveness in treatment of several diseases. Flavonoids can inhibit the secretion of inflammatory mediators such as nitric oxide, interleukin XII and tumor necrosis factor alpha (Nijveldt et al., 2001; Ostrowska and Skrzydlewska, 2005; Rao et al., 2005). Some studies mentioned the reduction of oxidative reactions following the administration of flavonoids (Ostrowska and Skrzydlewska, 2005; Kurin et al., 2012). Moreover, it has been demonstrated the flavonoid compounds of the quince have strong antioxidant and immune-regulatory effects (Nijveldt et al., 2001). According to the growing prevalence of diabetes and to the importance of normal fertility in physical and mental health, the aim of this study was to evaluate the protective effects of quince leaves extract, as herbal antioxidant, on the structural and functional alterations of testicular tissue following induction of diabetes.

\section{MATERIAL AND METHODS}

Animal Procedure: The number of 70 adult male Wistar rats with (mean \pm SD) of body weight $194.3 \pm 4.34 \mathrm{~g}$ was used in this study. The animals were used for all testing, housed under a $12 \mathrm{hrs}$. light-dark cycle with room temperature of 23$25^{\circ} \mathrm{C}$ and had access to food and water ad libitum. All animal procedures used in this study, were approved by the University of Tabriz standards for human care and use of laboratory animals in accordance with the Research Ethical Committee of the Ministry of Health and Medical Education of Iran (adopted in April 17, 2006) based on the Helsinki Protocol (Helsinki, Finland, 1975).

Experimental Design: The animals were divided into seven experimental groups. Each group consisted of ten animals (two animals were considered for replacement in cases of animal loss in duration of study). At the end of study, eight rats were used for sampling. 1) Control group (Cont.): normal and healthy rats that did not receive any type of treatment; 2) Low dose extract administrated control group (Cont+Ext250) which received quince leaf extract with dose of 250 $\mathrm{mg} / \mathrm{kg}$ body weight for eight weeks; 3) High dose extract administrated control group (Cont+Ext500) which received quince leaf extract with dose of 500 $\mathrm{mg} / \mathrm{kg}$ body weight for eight weeks; 4) Diabetic group (Diab.): in this group, diabetes was induced and the animals were euthanized eight weeks after induction of diabetes; 5) Low dose extract administrated Diabetic group (Diab+Ext250): in this group, two weeks after induction of diabetes, quince leaf extract was administrated in low dose to diabetic animals for a period of eight weeks; 6) High dose extract administrated Diabetic group (Diab+Ext500): this group consists of diabetic rats which received high dose of quince leaf extract for eight weeks after induction of diabetes. 7) Metformin treated Diabetic group (Diab+Met): this group consists of diabetic rats which received metformin for eight weeks after induction of diabetes. The experimental period was performed two weeks after induction of diabetes.

Treatments and Chemicals: Streptozotocin, STZ, (Sigma, ST. Louis, MO, USA) was used for induction of diabetes. The STZ was dissolved in 0.1 $\mathrm{M}$ citrate sodium buffer $(p \mathrm{H} \mathrm{4.5)}$ and was injected intraperitoneally (45 mg/kg body weight) in overnight fasting animals. Diabetes was confirmed 48 hours after injection of STZ with an automated glucose analyzer device (Glucometer, On Call EZ, SD, USA). The animals with blood glucose levels above $200 \mathrm{mg} / \mathrm{dl}$ were considered diabetic and were used in this study (Venkateswaran and Pari, 2002). The animals of group 7 , received daily freshly prepared metformin HCL (GLUCOPHAGE, Merck Sante s.a.s., LYON - FRANCE) dissolved in distilled water (100 mg/kg b.w.) by oral gavages method from two weeks after induction of diabetes to the end of study (Yanardag et al., 2005). The quince leaf extract was prepared according to Rodrigues et al method (Rodrigues et al., 2002).

Measurement of Body and Testicular Weight: At the beginning of study, the weight of each animal was recorded and then, the mean of body weight of experimental groups was recorded twice a week during the study. At the end of study, the animals were euthanized and the weight of right and left testes were measured.

Tissue Preparation and Histological Techniques: The testicular tissues were immediately fixed in 10\% formaldehyde in buffered solution containing $54 \mathrm{mM} \mathrm{NaH2PO} 4$ and 28 $\mathrm{mM} \mathrm{Na} 2 \mathrm{HPO} 4$ (pH 7.4) and kept at $4^{\circ} \mathrm{C}$. After 48 hours, the transverse section was made on the middle part of each testis and kept immersed in the fixative solution for the completion of tissue fixation. Then, formaldehyde-fixed samples were 
embedded in paraffin and sliced with thickness of 6-7 micrometer and were mounted onto albuminpre-coated glass slides. The mounted tissue samples were stained by the Hematoxylin and eosin method (H\&E) for histological observations by light microscopy.

Morphometric Analysis: For morphometric assessment of seminiferous tubules, the slides were studied at $200 \times$ magnification. To get extra precise results, only the seminiferous tubules (STs) that sectioned transversely were studied and the shortest diameter of seminiferous tubules was considered for measurement. For every one animal, three microscopic slides from different parts of testicular tissue were prepared and 200 tubules were studied.

Evaluation ofSpermatogenesisin Testicular Tissue: For estimation of spermatogenesis in testicular tissue, three different indices were used. Tubular differentiation index (TDI), repopulation index (RI) and spermiogenesis index (SPI). To determine the tubular differentiation index, the number of seminiferous tubules with more than three layers of germinal cells derived from type A of spermatogonia was calculated. To find out the repopulation index, the ratio of active spermatogonia (with euchromatic nucleus) to inactive ones (with dense heterochromatic nucleus) was evaluated. The ratio of the number of seminiferous tubules with spermatozoids to the empty tubules, were calculated for spermiogenesis index (Shetty et al., 2000; Meistrich et al., 2003).

Statistical Analysis: The obtained results were analyzed using the GraphPad PRISM ${ }^{\circledR}$ software version 5.04 (GraphPad Software, Inc. USA). All data were reported as mean \pm SD. The comparison of means between experimental groups was evaluated by using the one way analysis of variance (ANOVA) followed by Tukey's multiple comparison tests. Differences were considered to be statistically significant if $\mathrm{P}<0.05$.

\section{RESULTS AND DISCUSSION}

Blood Glucose: Induction of diabetes was led to significantincrease of fasting blood glucoselevels $(\mathrm{p}<0.0001)$ in all diabetic groups in comparison to control groups (Tab. 1). In this regard, treatment of diabetic groups with metformin was led to significant reduction of fasting blood glucose $(p<0.0001)$ in comparison to other diabetic groups. Moreover, the administration of quince leaf extract to control groups was not led to any noteworthy change in blood glucose levels.

Body Weight and Testicular Weight: Table 1 shows the mean of body weight and testicular weight in experimental groups. The administration of quince leaf extract, dose dependently was lead to decrement of body weight in control groups. In contrast, the mean of body weight was increased in extract treated diabetic rats compared to nontreated diabetic rats. The highest weight loss was observed in metformin treated diabetic group. The mean of body weight in control and Cont+Ext250 groups was significantly higher than all diabetic groups $(p<0.001)$. Figure 1 shows the changes of body weight from the beginning to the end of study. As table 1 shows, the mean of testicular weight was reduced significantly in

Tab. 1. Mean of body and testicular weight and fasting blood glucose levels in experimental groups

\begin{tabular}{lccc}
\hline & Body Weight (g) & Testicular Weight (g) & $\begin{array}{c}\text { Blood Glucose } \\
\text { (mg/d) }\end{array}$ \\
\hline Cont. & $206.1 \pm 14.61^{*}$ & $2.325 \pm 0.19^{*}$ & $96.50 \pm 7.52^{*}$ \\
\hline Cont+Ext250 & $203.3 \pm 7.05^{*}$ & $2.213 \pm 0.19^{*}$ & $96.88 \pm 9.04^{*}$ \\
\hline Cont+Ext500 & $194.3 \pm 4.51$ & $2.20 \pm 0.22^{*}$ & $92.75 \pm 13.60^{*}$ \\
\hline Diab. & $180.3 \pm 19.98$ & $1.87 \pm 0.13$ & $308 \pm 49.94$ \\
\hline Diab+Ext250 & $181.6 \pm 13.65$ & $1.81 \pm 0.18$ & $253.4 \pm 57.02$ \\
\hline Diab+Ext500 & $184.7 \pm 10.98$ & $1.75 \pm 0.11$ & $244.3 \pm 73.91$ \\
\hline Diab+Met & $179.5 \pm 22.10$ & $1.70 \pm 0.15$ & $169 \pm 33.20^{\beta}$ \\
\hline
\end{tabular}

Note: Body and testicular weight: " Significant difference in comparison to all diabetic groups. Blood glucose: *Significant difference in comparison to all diabetic groups; ${ }^{\beta}$ Significant difference in comparison to diabetic groups. Data are presented as mean \pm SD. 
diabetic groups in comparison to control groups ( $\mathrm{p}<0.001$ ). In diabetic groups, the most reduction of testicular weight was observed in metformin treated diabetic group.

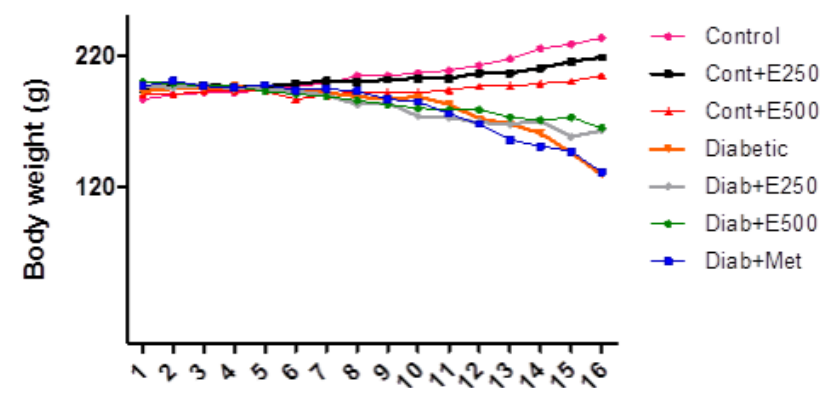

Fig. 1. Diagram of body weight gain during the period of study. The body weight measurement was carried out twice a week.

Cellular and Morphometric Analysis of Seminiferous Tubules: The mean of external diameter of seminiferous tubules was reduced slightly in control groups following administration of quince leaf extract (Tab. 2). In diabetic groups, the administration of quince leaf extract was lead to increase of external diameter of STs but these changes were not significant. This index was reduced significantly in diabetic groups in comparison to control groups $(\mathrm{p}<0.001)$. There was no significant difference between metformin treated diabetic group and extract treated control groups. As table 2 shows, the mean of internal diameter of STs was reduced in diabetic groups but this change was not significant. Following induction of diabetes the mean of germinal epithelium height was reduced in diabetic groups in comparison to control group ( $p<0.001)$. Treatment of diabetic rats with quince leaf extract was lead to non-significant increase of height of germinal epithelium. Significant difference was observed in mean of germinal epithelium height between three diabetic groups and quince leaf treated control groups. Thickness of testicular capsule was increased in diabetic groups in comparison to control groups. In this regard, the most thickness of testicular capsule was observed in non-treated diabetic group (Tab. 2).

The mean of cellular population of STs Wall was summarized in table 3. Accordingly, the number of normal Sertoli cells was reduced in quince leaf extract control groups. The number of Sertoli cells was reduced significantly in non-treated diabetic and extract treated diabetic groups in comparison to control and Cont+Ext250 groups. Treatment of diabetic rats with metformin was lead to significant increment of Sertoli cells in comparison to other diabetic groups $(\mathrm{p}<0.001)$. The mean of number of spermatogonia was not changed significantly in experimental groups. The number of primary spermatocytes was reduced in diabetic groups. In this regard, the administration of quince leaf extract dose dependently led to increase of primary spermatocytes in diabetic groups. In metformin treated diabetic rats the number of primary spermatocytes was significantly higher than non-treated diabetic group. The mean of number of round spermatids significantly reduced in diabetic groups in comparison to control groups $(p<0.001)$. Treatment of diabetic rats with quince

Tab. 2. Morphometric analysis indices in experimental groups.

\begin{tabular}{lcccc}
\hline & $\begin{array}{c}\text { External } \\
\text { Diameter }(\mu \mathrm{m})\end{array}$ & $\begin{array}{c}\text { Internal } \\
\text { Diameter }(\mu \mathrm{m})\end{array}$ & $\begin{array}{c}\text { Germinal Epithelium } \\
\text { height }(\mu \mathrm{m})\end{array}$ & $\begin{array}{c}\text { Capsular } \\
\text { Thickness }(\mu \mathrm{m})\end{array}$ \\
\hline Cont. & $429.8 \pm 33.27^{*}$ & $282.3 \pm 32.95$ & $141.5 \pm 14.75^{*}$ & $58.30 \pm 7.21$ \\
\hline Cont+Ext250 & $413.2 \pm 24.52^{\beta}$ & $280.7 \pm 30.69$ & $135.6 \pm 10.29^{\beta}$ & $56.10 \pm 6.59^{*}$ \\
\hline Cont+Ext500 & $415.5 \pm 17.98^{\beta}$ & $275.7 \pm 21.26$ & $136.7 \pm 11.36^{\beta}$ & $57.50 \pm 6.41$ \\
\hline Diab. & $369.5 \pm 44.19$ & $257.9 \pm 50.16$ & $109.2 \pm 20.28$ & $67.40 \pm 9.16$ \\
\hline Diab+Ext250 & $372.6 \pm 32.68$ & $257.7 \pm 38.79$ & $115 \pm 14.41$ & $62 \pm 6.92$ \\
\hline Diab+Ext500 & $375.1 \pm 34.39$ & $260 \pm 43.31$ & $118 \pm 16.52$ & $61.40 \pm 9.44$ \\
\hline Diab+Met & $394.8 \pm 26.15$ & $275.9 \pm 24.62$ & $122.3 \pm 19.27$ & $60 \pm 6.89$ \\
\hline
\end{tabular}

Note: External Diameter \& Germinal epithelium: ${ }^{*}$ Significant difference in comparison to all diabetic groups; ${ }^{\beta}$ Significant difference in comparison to all diabetic groups except metformin treated diabetic group. Capsular thickness: ${ }^{*}$ Significant difference in comparison to diabetic group. Data are presented as mean \pm SD. 
Tab. 3. Cellular population of germinal epithelium of seminiferous tubules in experimental groups.

\begin{tabular}{lcccc}
\hline & $\begin{array}{c}\text { Sertoli } \\
\text { (\#cell/10tubules) }\end{array}$ & $\begin{array}{c}\text { Spermatogonia } \\
\text { (\#cell/10tubules) }\end{array}$ & $\begin{array}{c}\text { Primary } \\
\text { Spermatocyte } \\
\text { (\#cell/10tubules) }\end{array}$ & $\begin{array}{c}\text { Spermatids } \\
\text { (\#cell/10tubules) }\end{array}$ \\
\hline Cont. & $429.8 \pm 33.27^{*}$ & $282.3 \pm 32.95$ & $141.5 \pm 14.75^{*}$ & $58.30 \pm 7.21^{*}$ \\
\hline Cont+Ext250 & $413.2 \pm 24.52^{*}$ & $280.7 \pm 30.69$ & $135.6 \pm 10.29^{\beta}$ & $56.10 \pm 6.59^{*}$ \\
\hline Cont+Ext500 & $415.5 \pm 17.98^{\beta}$ & $275.7 \pm 21.26$ & $136.7 \pm 11.36^{\beta}$ & $57.50 \pm 6.41^{*}$ \\
\hline Diab. & $369.5 \pm 44.19$ & $257.9 \pm 50.16$ & $109.2 \pm 20.28$ & $67.40 \pm 9.16$ \\
\hline Diab+Ext250 & $372.6 \pm 32.68$ & $257.7 \pm 38.79$ & $115 \pm 14.41$ & $62 \pm 6.92$ \\
\hline Diab+Ext500 & $375.1 \pm 34.39$ & $260 \pm 43.31$ & $118 \pm 16.52$ & $61.40 \pm 9.44$ \\
\hline Diab+Met & $394.8 \pm 26.15^{\alpha}$ & $275.9 \pm 24.62$ & $122.3 \pm 19.27^{\alpha}$ & $60 \pm 6.89^{\alpha}$ \\
\hline
\end{tabular}

Note: Sertoli cells: *Significant difference in comparison to all diabetic groups except metformin treated diabetic group; ${ }^{\beta}$ Significant difference in comparison to Diab. and Diab+Ext500 groups; ${ }^{\alpha}$ Significant difference in comparison to diabetic groups. Primary Spermatocyte: Significant difference in comparison to all diabetic groups; ${ }^{\beta}$ Significant difference in comparison to all diabetic groups except metformin treated diabetic group; ${ }^{\alpha}$ Significant difference in comparison to diabetic group. Spermatids: ${ }^{*}$ Significant difference in comparison to all diabetic groups; ${ }^{\alpha}$ Significant difference in comparison to diabetic group. Data are presented as mean \pm SD.

leaf extract led to increase of the number of spermatids. In metformin treated diabetic group, the number of spermatids was significantly higher compared to non-treated diabetic group.

Microscopic Indices of Spermatogenesis: Tubular differentiation index (TDI) was reduced in diabetic groups in comparison to control groups (Fig. 2a). Moreover, treatment of diabetic rats with quince leaf extract led to increase of this index in comparison to non-treated diabetic rats. In metformin treated diabetic group, this index was non-significantly higher than other diabetic groups. The index of spermiogenesis (SPI) was higher in control groups in comparison to diabetic groups (Fig. 2b). In diabetic groups, treatment with quince leaf extract was led to improvement of this index but, this difference was not significant ( $p$ $>0.05$ ). According to figure $2 c$, the most reduction of repopulation index (RI) was observed in nontreated diabetic group. Moreover, dose dependent administration of quince leaf extract to diabetic groups led to elevation of this index in comparison to non-treated diabetic rats.

Fig. 2. Microscopic indices of spermatogenesis. 2a and 2b: ${ }^{\alpha}$ Significant difference in comparison to diabetic group. 2c: ${ }^{\alpha}$ Significant difference in comparison to diabetic group; ${ }^{\beta}$ Significant difference in comparison to Diab+E250 group; "Significant difference in comparison to Diab+Met group. Data are presented as mean \pm SD.
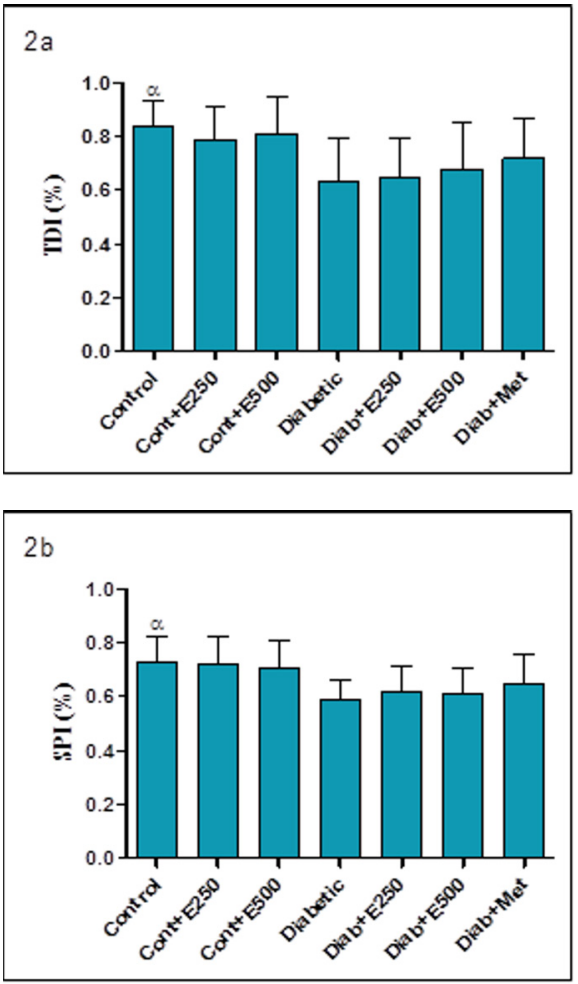

$2 c$

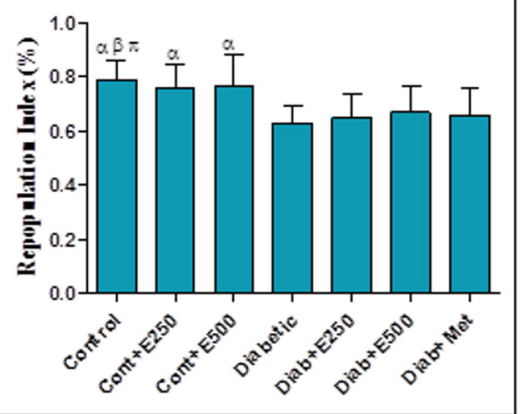


Histology of Testicular Tissue: Figure 3 shows the histologic structure of testicular tissue in experimental groups. Tubular atrophy, disarrangement of germinal epithelium and depopulation of spermatogenic cells was the most visible changes observed in diabetic groups. Moreover, above mentioned changes were reduced in quince leaf extract treated diabetic rats.
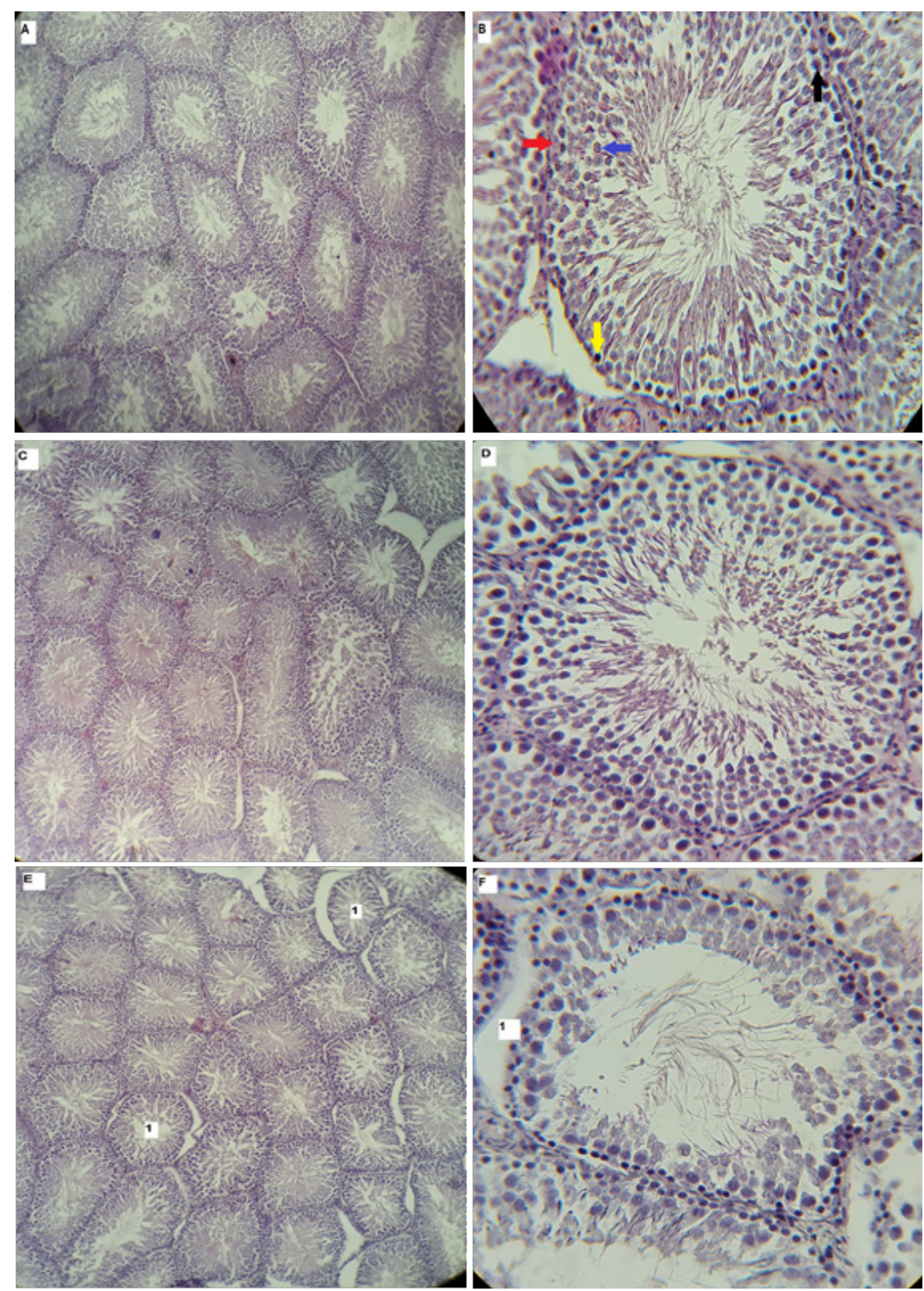

Fig. 3. Histologic structure of testicular tissue in experimental groups. A, B: Control group. General structure of STs with normal architecture (A); structure of normal ST with higher magnification (B). C, D: Cont+Ext250 group. The structure of STs and germinal epithelium are similar to control group. E, F: Cont+Ext500 group. Slight tubular atrophy is visible (1). G, H: Diabetic group. Increase of capsular thickness (2) and malformation of STs (3) is visible (G); reduction of spermatogenic cells (4) is visible (H). I, J: Diab+Ext250 group. Tubular atrophy (5) is visible (I); germinal epithelium population is higher than diabetic group (J). K, L: Diab+Ext500 group. Tubular atrophy (6) still visible (K); disarrangement and depopulation of germinal epithelium considerably decreased in this group (L). M, N: Diab+Met group. Mild tubular atrophy (7) is visible in some STs (M); arrangement and populace of spermatogenic cells somewhat is similar to control group (N). Red arrow: Sertoli cell; Yellow arrow: Spermatogonia; Black arrow: Spermatocyte; Blue arrow: Spermatid. (Hematoxylin \& Eosin stain; A,C,E,G,I,K,M: $100 \times ; B, D, F, H, J, L, N: 400 \times$ ) 

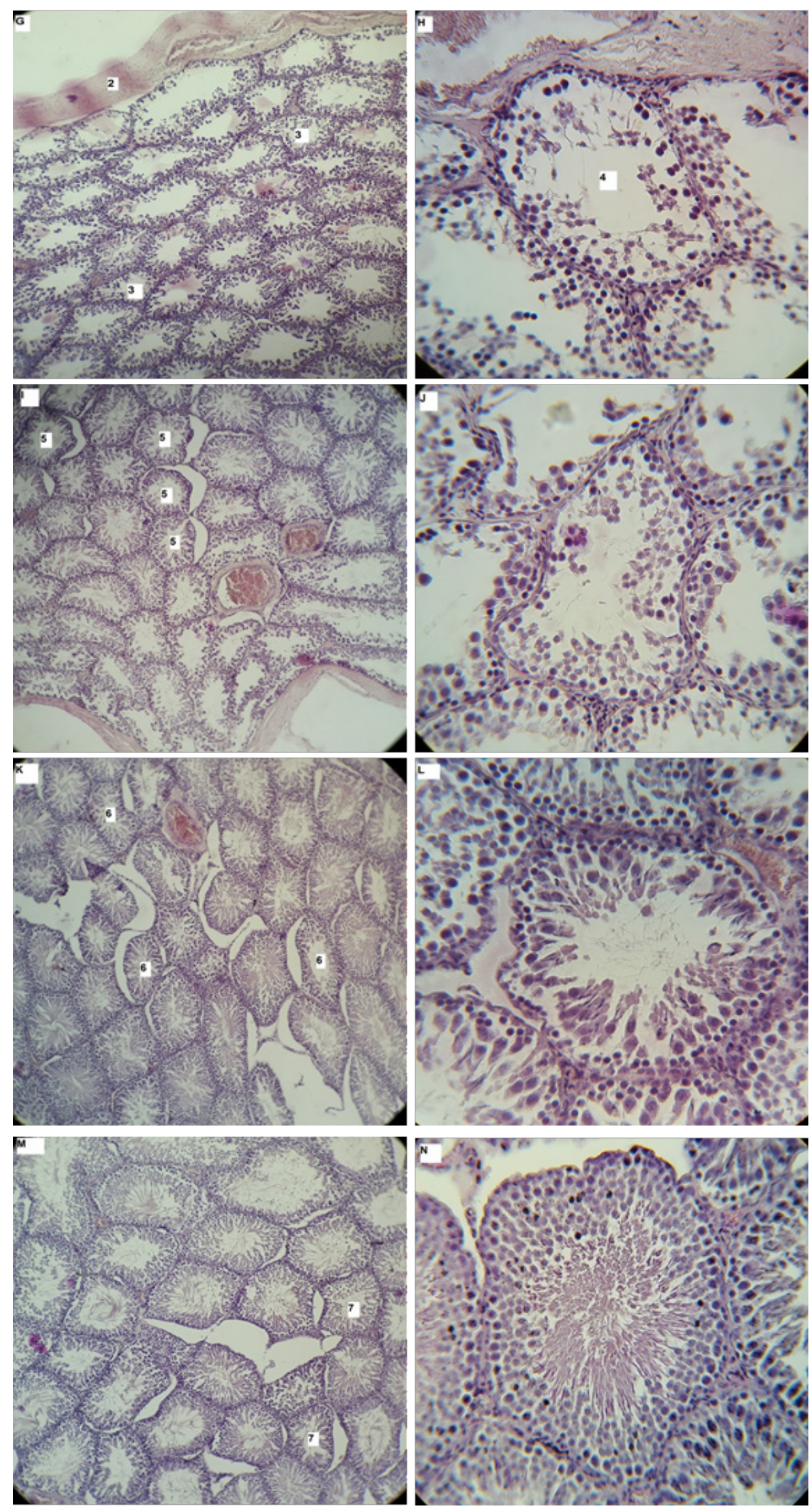
In this study the protective effects of quince leaf extract on the microscopic alterations of testicular tissue following induction of diabetes were investigated. Diabetic complications are the major problems that occur in diabetic patients (Yanardag et al., 2005). Oxidative stress and production of reactive oxygen species (ROS) play a key role in the pathogenesis of diabetes mellitus and subsequently development of diabetes complications (Maritim et al., 2003; Giacco and Brownlee, 2010).

Induction of diabetes is associated with cellular alterations in testicular microenvironment (Kianifard et al., 2011). The Quince (cydonia oblonga) has a large amount of phenolic acids and flavonoids as antioxidant compounds (Silva et al., 2004; Oliveira et al., 2008; Magalhaes et al., 2009). Reaction of flavonoids with free radicals leads to formation of more stable radicals with lower cytotoxicity. As well, they are capable of chelating iron ions $(\mathrm{Fe} 2+)$ which lead to reduction of side effects of free radicals (Nijveldt et al., 2001). In this way, flavonoids can inhibit the secretion of inflammatory mediators such as nitric oxide, interleukin XII and tumor necrosis factor alpha (Nijveldt et al., 2001; Ostrowska and Skrzydlewska, 2005; Rao et al., 2005). Some studies mentioned the reduction of oxidative reactions following the administration of flavonoids (Ostrowska and Skrzydlewska, 2005; Kurin et al., 2012). It has been demonstrated that, flavonoid compounds of the quince have strong antioxidant and immuneregulatory effects (Nijveldt et al., 2001). Moreover, it has been demonstrated that the most phenolic contents have been found in leaves in comparison to other parts of this plant (Oliveira et al., 2007). Flavonoids are the classes of compounds that have antidiabetic properties. Intake of flavonoids might prove to be important for alternative diabetes treatments or reduction of the risk of the disease (Coman et al., 2012). In the present study, the mean of blood glucose was reduced in extract treated diabetic rats. Various phenolic compounds such as kaempferol have been reported for cydonia oblonga (Oliveira et al., 2008). It has been found that kaempferol molecules protected the cells against the apoptosis induced by oxidative stress (Lee et al., 2010). As a result, the flavonoid compounds through reducing of the pancreatic $\beta$-cell death can be consider as one of the possible mechanisms in cases of reduction of blood glucose in quince leaf extract treated diabetic rats. According to our results, it seems that the ability of quince leaf extract in reducing of blood glucose levels is lower than metformin. In this regard, several factors such as the root of administration, the period of administration and the effective dose of extract can affect the hypoglycemic effects of quince leaf extract in comparison to metformin. In this study, treatment of control groups with quince leaf extract was lead to slight decrease of body weight while, in quince leaf extract treated diabetic groups increase of body weight were observed. According to figure 1, it seems that, diabetes related weight loss process is reduced following treatment of diabetic rats with quince leaf extract. The reduction of body weight can be due to breakdown of tissue proteins (Andulla and Varadacharyulu, 2003; Yanardag et al., 2005). Diminution of pancreatic $\beta$-cells depopulation and improvement of glucose metabolism could be effective in weight gain of diabetic rats following the administration of quince leaf extract. It seems that, the changes of gonadal weight somewhat are similar to the changes of body weight. Our study is the first in-vivo report about the effects of quince leaf extract on the alterations of testicular tissue in diabetic conditions.

Excessive production of reactive oxygen species and microvascular damage are reported to substantially contribute to the occurrence of structural and functional changes (Nishikawa and Araki, 2007; Shrilatha and Muralidhara, 2007; Aitken and Roman, 2008). Disruption of spermatogenesis in diabetic rats was reported in various studies (Cai et al., 2000; Guneli et al., 2008; Kianifard and Rezaee, 2015). According to our results, in diabetic rats decrease in diameter of STs was accompanied with depletion in the height of germinal epithelium. This finding indicates that, the STs became atrophied during the course of diabetes. These histological observations in STs, illustrated depressed cellular activity of spermatogenic cells. Oxidative stress in testicular tissue has a direct relationship with abnormal spermatogenesis due to decrement of glutathione in male germ cells which leads to incomplete functional maturation and capacitation of spermatozoa (Maiorino and Ursini, 2005; Reddy et al., 2006). Our study showed that, the administration of quince leaf extract, leads to 
improvement of histomorphometry of testicular tissue in diabetic groups.

In our study, the number of normal sertoli cells (normal nuclear shape and position of cell) decreased in diabetic groups. This reduction could be due to depopulation of germ cells. In diabetic rats, the number of primary spermatocytes decreased significantly in comparison to control groups. This finding indicates that, the conversion of spermatogonia to primary spermatocytes is reduced in diabetic conditions.

The evaluation of microscopic indices of spermatogenesis confirms these histologic results. Diminished tubular differentiation (TDI) and spermiogenesis (SPI) indices in diabetic rats indicates that, conversion of spermatogonia to primary spermatocytes is reduced. Reduction of repopulation index in diabetic rats demonstrates the number of inactive spermatogonia increased after induction of diabetes. This process causes a decline of the number of primary spermatocytes derived from spermatogonia cells. These alterations in cellular conversion and/or activity lead to reduction in production of spermatozoids. It seems that, prevention of oxidative damage with natural antioxidants can be considered as one of the most effective strategies in control and reduction of diabetic complications in laboratory animals. The results obtained in this study, are valid for experimental diabetes and cannot be extrapolated automatically to human diabetes.

\section{CONCLUSION}

According to the results of this study, it seems that, quince leaf extract may play a positive role in improvement of microscopic structure of testicular tissue in diabetic conditions due to its antioxidant properties.

Acknowledgments: This work was financially supported by research council of University of Tabriz.

\section{REFERENCES}

1. Aitken RJ, Roman SD (2008). Antioxidant systems and oxidative stress in the testes. Oxid Med Cell Longev 1:1524.

2. Andulla B, Varadacharyulu NCh (2003). Antioxidant role of mulberry leaves in streptozotocin-diabetic rats. Clin Chim Acta 338:3-10.

3. Cai L, Chen S, Evans T, Xi Deng D, Mukherjee K, Chakrabarti S (2000). Apoptotic germ-cell death and testicular damage in experimental diabetes: prevention by endothelin antagonism. Urol Res 28:342-347.
4. Coman C, Rugina OD, Socaciu C (2012). Plants and Natural Compounds with Antidiabetic Action. Not Bot Horti Agrobo 40(1):314-325.

5. Giacco F, Brownlee M (2010). Oxidative stress and diabetic complications. Circ Res 107:1058-1070.

6. Guneli E, Tugyan K, Ozturk H, Gumustekin M, Cilaker S, Uysal N (2008). Effect of melatonin on testicular damage in streptozotocin-induced diabetes rats. Eur Surg Res 40:354-360.

7. Kianifard D, Sadrkhanlou RA, Hasanzadeh S (2011). The Ultrastructural Changes of the Sertoli and Leydig Cells Following Streptozotocin Induced Diabetes. Iran J Bas Med Sci 15:623-635.

8. Kianifard D, Rezaee F (2015). Beneficial Effects of Coenzyme Q 10 in Reduction of Testicular Tissue Alteration Following Induction of Diabetes in Adult Rats. Roman J Diab Nut Metab Dis 22(1):019-027.

9. Kurin E, Atanasov AG, Donath O, Heiss EH, Dirsch VM, Nagy M (2012). Synergy study of the inhibitory potential of red wine polyphenols on vascular smooth muscle cell proliferation. Planta Medica 78(8):772-778.

10. Lee JL, Suh KS, Choi MC, Chon S, Oh S, Woo JT, Kim SW, Kim JW, Kim YS (2010). Kaempferol protects HIT-15 pancreatic beta-cells from 2-deoxy-D-ribose-induced oxidative damage. Phytoter Res 24:419-423.

11. Magalhaes AS, Silva BM, Pereira JA, Andrade PB, Valentao P, Carvalho M (2009). Protective effect of quince (Cydonia oblonga Miller) fruit against oxidative hemolysis of human erythrocytes. Food Chem Toxicol 47(6):1372-1377.

12. Maiorino M, Ursini F (2005). Oxidative stress, spermatogenesis and fertility. Biol Chem 383:591-597.

13. Maritim AC, Sanders RA, Watkins JB (2003). Diabetes, oxidative stress, and antioxidants: a review. J Biochem Mol Toxicol 17:24-38.

14. Meistrich ML, Wilson G, Porter KL, Huhtaniemi I, Shetty G, Shuttlesworth GA (2003). Restoration of spermatogenesis in dibromochloropropane (DBCP)-treated rats by hormone suppression. Toxicol Sci 76(2): 418-426.

15. Nijveldt RJ, Van Nood E, Van Hoorn DE, Boelens PG, Van Norren K, Van Leeuwen PA (2001). Flavonoids: a review of probable mechanisms of action and potential applications. Americ J Clin Nut 74(4):418-425.

16. Nishikawa T, Araki E (2007). Impact of mitochondrial ROS production in the pathogenesis of diabetes mellitus and its complications. Antioxid Redox Signal 9:343-353.

17. Oliveira AP, Pereira JA, Andrade PB, Valento P, Seabra RM, Silva BM (2007). Phenolic profile of Cydonia oblonga Miller leaves. J Agri Food Chem 55:7926-7930.

18. Oliveira AP, Pereira JA, Andrade PB, Valentão P, Seabra RM, Silva BM (2008). Organic acids composition of Cydonia oblonga Miller leaf. Food Chem 111(2):393-399.

19. Orth JM, Murray FT, Bardin CW (1979). Ultrastructural changes in Leydig cells of streptozotocin-induced diabetic rats. Anat Rec 195:415-430.

20. Ostrowska J, Skrzydlewska E (2005). The comparison of effect of catechins and green tea extract on oxidative modification of LDL in vitro. Advanc Med Sci 51:298-303. 
21. Rao YK, Fang SH, Tzeng YM (2005). Inhibitory Effects of the Flavonoids Isolated from Waltheria indica on the Production of NO, TNF-ALPHA and IL-12 in Activated Macrophages. Biol Pharmaceut Bull 28(5):912-915.

22. Reddy MM, Mahipal SV, Subhashini J, Reddy MC, Roy KR, Reddy GV, Reddy PR, Reddanna P (2006). Bacterial lipopolysaccharide-induced oxidative stress in the impairment of steroidogenesis and spermatogenesis in rats. Reprod Toxicol 22:493-500.

23. Rodrigues AL, da Silva GL, Mateussi AS, Fernandes ES, Miguel OG, Yunes RA, Calixto JB, Santos AR (2002). Involvement of monoaminergic system in the antidepressant-like effect of the hydroalcoholic extract of Siphocampylus verticillatus. Life Sci 70(12):1347-1358.

24. Shetty G, Wilson G, Huhtaniemi I, Shuttlesworth GA, Reissmann T, Meistrich ML (2000). Gonadotropin releasing hormone analogs and testosterone inhibits the recovery of spermatogenesis in irradiated rats. Endocrinol 141:1735-1745.

25. Shrilatha B, Muralidhara (2007). Early oxidative stress in testis and epididymal sperm in streptozotocininduced diabetic mice: its progression and genotoxic consequences. Reprod Toxicol 23:578-587.

26. Silva BM, Andrade PB, Valentao P, Ferreres F, Seabra RM, Ferreira MA (2004). Quince (Cydonia oblonga Miller) fruit (pulp, peel, and seed) and jam: antioxidant activity. J Agri Food Chem 52(15):4705-4712.

27. Venkateswaran S, Pari L (2002). Antioxidant effect of Phaseolus vulgaris in streptozotocin-induced diabetic rats. Asia Pac J Clin Nutr 11:206-209.

28. Yanardag R, Ozsoy-Sacan O, Bolkent S, Orak H, KarabulutBulan $O$ (2005). Protective effects of metformin treatment on the liver injury of streptozotocin-diabetic rats. Hum Exp Toxicol 24:129-135. 